\title{
Microstructural Changes of the Baboon Cerebral Cortex during Gestational Development Reflected in Magnetic Resonance Imaging Diffusion Anisotropy
}

\author{
Christopher D. Kroenke, ${ }^{1}$ David C. Van Essen, ${ }^{2}$ Terrie E. Inder, ${ }^{3,4,5}$ Sandra Rees, ${ }^{6}$ G. Larry Bretthorst,${ }^{5}$ and \\ Jeffrey J. Neil $3,4,5$ \\ ${ }^{1}$ Advanced Imaging Research Center, Oregon National Primate Research Center, and Department of Behavioral Neuroscience, Oregon Health and Science \\ University, Portland, Oregon 97239, Departments of ${ }^{2}$ Anatomy and Neurobiology, ${ }^{3}$ Pediatrics, ${ }^{4}$ Pediatric Neurology, and ${ }^{5}$ Radiology, Washington \\ University School of Medicine, St. Louis, Missouri 63110, and ${ }^{6}$ Department of Anatomy and Cell Biology, University of Melbourne, Victoria, 3010, Australia
}

Cerebral cortical development involves complex changes in cellular architecture and connectivity that occur at regionally varying rates. Using diffusion tensor magnetic resonance imaging (DTI) to analyze cortical microstructure, previous studies have shown that cortical maturation is associated with a progressive decline in water diffusion anisotropy. We applied high-resolution DTI to fixed postmortem fetal baboon brains and characterized regional changes in diffusion anisotropy using surface-based visualization methods. Anisotropy values vary within the thickness of the cortical sheet, being higher in superficial layers. At a regional level, anisotropy at embryonic day 90 (E90; 0.5 term; gestation lasts $185 \mathrm{~d}$ in this species) is low in allocortical and periallocortical regions near the frontotemporal junction and is uniformly high throughout isocortex. At E125 (0.66 term), regions having relatively low anisotropy (greater maturity) include cortex in and near the Sylvian fissure and the precentral gyrus. By E146 (0.8 term), cortical anisotropy values are uniformly low and show less regional variation. Expansion of cortical surface area does not occur uniformly in all regions. Measured using surface-based methods, cortical expansion over E125-E146 was larger in parietal, medial occipital, and lateral frontal regions than in inferior temporal, lateral occipital, and orbitofrontal regions. However, the overall correlation between the degree of cortical expansion and cortical anisotropy is modest. These results extend our understanding of cortical development revealed by histologic methods. The approach presented here can be applied in vivo to the study of normal brain development and its disruption in human infants and experimental animal models.

Key words: brain development; nonhuman primate; cortical surface; diffusion; prenatal; MRI

\section{Introduction}

Cerebral cortical development in primates is a complex process that occurs over an extended period, mainly during the second half of gestation. Regional differences have been reported in the timing of major events, including neuronal proliferation near the ventricular margins, migration to the cortical plate, formation of cortical layers, elaboration of local dendritic and axonal arbors, establishment of long-distance connections, and formation of gyral and sulcal folds (Conel, 1939; Sidman and Rakic, 1982; Smart, 1983; Brody et al., 1987; Kinney et al., 1988; Kostovic et al., 2002). However, many aspects of cortical differentiation have been difficult to quantify using conventional anatomical meth-

\footnotetext{
Received July 6, 2007; revised Aug. 31, 2007; accepted Sept. 19, 2007.

This work was supported by National Institutes of Health Grants NS37357, CA83060, and NS43010, HL52636 from the Bronchopulmonary Dysplasia Resource Center, and P51RR13986 for facility support, Grant MH-60974 (funded by the National Institute of Mental Health, National Institute of Biomedical Imaging and Bioengineering, Nationa Science Foundation), the Murdoch Children's Research Institute, and the Allen P. and Josephine B. Green fund for Pediatric Neurology. We thank Dr. Joel Price and Dr. Michelle Loeliger for useful discussions and John Harwell and Donna Dierker for Caret software development. We acknowledge the excellent technical assistance of Sarah Cain in preparing the histological sections.

Correspondence should be addressed to Dr. Jeff Neil, Pediatric Neurology, St. Louis Children's Hospital, One Children's Place, St. Louis, M0 63110. E-mail: neil@wustl.edu.

DOI:10.1523/JNEUROSCI.3063-07.2007

Copyright $\odot$ 2007 Society for Neuroscience $\quad 0270-6474 / 07 / 2712506-10 \$ 15.00 / 0$
}

ods. Here, we analyze cortical maturation quantitatively and with high spatial resolution in the immature baboon brain using (1) diffusion tensor imaging (DTI), a type of magnetic resonance imaging (MRI) that reveals anisotropy in local cellular microstructure, and (2) surface based measures of cortical expansion.

High water diffusion anisotropy occurs in regions where cellular processes (axons, dendrites, glial processes) are locally ordered on a millimeter scale and reflects greater water displacement parallel to the longitudinal axis of cellular processes than transverse to them. Early in cortical development, the radial organization of pyramidal and glial cells gives rise to directionally dependent (i.e., anisotropic) diffusion (McKinstry et al., 2002). The physical relationship between diffusion MRI measurements and cellular scale cortical microarchitecture is under active investigation (Kroenke et al., 2004; Jespersen et al., 2007). Empirically, radial diffusion anisotropy progressively declines throughout cortical maturation (McKinstry et al., 2002; Deipolyi et al., 2005) in parallel with elaboration of dendritic and axonal arbors throughout the cortical sheet. In contrast to the pattern in cerebral cortex, diffusion anisotropy increases with maturation in white matter (Huppi et al., 1998; Neil et al., 1998).

Regional differences in cortical diffusion anisotropy have been reported in prematurely born human infants (Deipolyi et 
Table 1. Brains evaluated by MRI

\begin{tabular}{|c|c|c|c|c|c|c|c|c|}
\hline Brain & Hemisphere & Sex & $\begin{array}{l}\text { Image } \\
\text { resolution } \\
(\mathrm{mm})\end{array}$ & $\mathrm{T}_{2}(\mathrm{TE} / \mathrm{TR} / \mathrm{NEX})^{a}$ & Diffusion (TE/TR/NEX) ${ }^{a}$ & $\begin{array}{l}\text { Total } \\
\text { time } \\
\text { (hours) }\end{array}$ & Fixative $^{c}$ & $\begin{array}{l}\text { Fixation } \\
\text { interval } \\
\text { (months) }\end{array}$ \\
\hline E90 & Left & $M$ & 0.35 & $0.14 / 5.5 / 12$ & $0.067 / 4 / 8$ & 61 & Formalin & 37 \\
\hline E125a & Left & $\mathrm{F}$ & 0.5 & $0.1 / 5.5 / 8$ & $0.067 / 3.45 / 6$ & 40 & $4 \%$ PFA & 9 \\
\hline $\mathrm{E} 125 \mathrm{~b}$ & Left & $\mathrm{F}$ & 0.5 & $0.1 / 3.5 / 32$ & $0.067 / 4 / 8$ & 66 & Formalin & 45 \\
\hline $\mathrm{E} 125 \mathrm{C}$ & Right & $M$ & 0.5 & $0.12 / 4.5 / 8$ & $0.067 / 4 / 6$ & 43 & Formalin & 22 \\
\hline E146 & Right & M & 0.5 & $0.1 / 5 / 12$ & $0.067 / 4 / 8$ & 65 & Formalin & 38 \\
\hline E175 & $\mathrm{N} / \mathrm{A}^{b}$ & $\mathrm{~F}$ & 0.5 & $0.1 / 4.5 / 12$ & $0.067 / 4 / 8$ & 61 & $4 \%$ PFA & 12 \\
\hline E185 & $\mathrm{N} / \mathrm{A}^{b}$ & M & 0.5 & $0.1 / 5 / 16$ & $0.067 / 4 / 6$ & 54 & $4 \%$ PFA & 13 \\
\hline
\end{tabular}

${ }^{a}$ Echo time (TE) and repetition time (TR) are in units of seconds. NEX, is the number of excitations used for MR signal averaging.

${ }^{b}$ Cortical surfaces of brains E175 and E185 were not generated; N/A, not applicable.

'Formalin, 10\% formalin; 4\% PFA, 4\% phosphate-buffered paraformaldehyde.

al., 2005) and in developing baboon brain analyzed postmortem (Kroenke et al., 2005), but the underlying biological source of this variation has not been described. These previous studies analyzed variation between limited regions of interest or within individual MRI slices. Here, we use cortical surface modeling procedures (Van Essen et al., 2001) to characterize diffusion anisotropy throughout the developing cerebral cortex. This analysis reveals similarities between cortical anisotropy patterns and (1) the boundary between allocortex and isocortex, (Zilles, 2004), (2) a previously described gradient in neurogenesis and subsequent cortical maturation emanating from the insula (Sidman and Rakic, 1982; Smart, 1983; Smart et al., 2002), and (3) maturational differences between primary sensory and motor areas versus nonprimary cortical areas (Conel, 1939; Sidman and Rakic, 1982; Brody et al., 1987; Kinney et al., 1988; Kostovic et al., 2002).

Surface-based procedures also enable examination of two additional aspects of cortical development. The first relates to differences that may exist in anisotropy between superficial and deep cortical layers. Given the inside-out pattern of cortical development (Rakic, 1995), diffusion anisotropy may be anticipated to be higher in superficial compared with deep layers (Sizonenko et al., 2007). Second, measurements of cortical surface expansion are presented to provide an assessment of regional variation in the expansion rate and potential correlation between regional patterns of expansion and reductions in cortical diffusion anisotropy.

\section{Materials and Methods}

MRI data acquisition. Animal husbandry was performed at the Southwest Foundation for Biomedical Research (San Antonio, TX). The immature baboons were delivered at known gestation by cesarean section. After delivery they were immediately killed with a high dose of phenobarbitol followed by immersion fixation of the brain in 10\% formalin (for necropsies performed before 2004, four samples) or $4 \%$ paraformaldehyde (necropsies 2005 and later, three samples). The brains were permanently stored in fixative. Table 1 specifies the fixative used for each brain, and the length of time that elapsed between necropsy and the MRI procedures. In addition to the brains listed in Table 1, we have collected diffusion MRI data on $\sim 50$ similarly prepared tissue specimens under various experimental settings (such as after induced delivery) (our unpublished observations). We have not observed consistent differences between formalin- and paraformaldehyde-fixed brains with our imaging methods. However, we cannot eliminate the possibility that subtle fixation effects are obscured by other factors such as fixation interval within our sample collection. Animal handling and ethics were approved to conform to American Association for Accreditation of Laboratory Animal Care guidelines. Procedures have been described previously (Kroenke et al., 2005). Table 1 summarizes data for seven brains analyzed by MRI.

The MRI experiments were performed on brains immersed in fixative. Brains embryonic day 90 (E90), E125b, and E125c were small enough to fit into 60-cc syringes, which were placed within a 5 -cm-diameter transmit/receive quadrature Litz radio frequency (RF) coil (Doty et al., 1999) (Doty Scientific, Columbia, SC). Brain E125a was scanned within a 6-cm-diameter rigid container filled with fixative. The remaining brains were sealed within plastic bags containing fixative, and the bags were placed within $6.8-\mathrm{cm}$ diameter cylindrical containers filled with a perfluorinated liquid (Krytox; DuPont, Deepwater, NJ). Brain E125a and those at later developmental stages were imaged using a $7-\mathrm{cm}$ diameter quadrature transmit/receive Litz RF coil. Given the similar designs of the RF coils used for this study, the signal-to-noise ratio per volume element scales approximately inversely with the coil volume. As a result, anisotropy measurements are more precise for brains E125b and E125c, in which voxel sizes of $0.5 \times 0.5 \times 0.5 \mathrm{~mm}$ were acquired using the 5 -cm-diameter coil than for the remaining brains. However, differences in precision are unlikely to affect our analyses because the regional and temporal patterns described herein were obtained from large numbers of voxels within each brain.

The MRI data were acquired in the axial plane using a multislice, spin-echo imaging sequence. Imaging was performed using a 4.7-T small animal imaging system consisting of an Oxford Instruments (Oxford, UK) magnet and a Varian (Palo Alto, CA) NMR Systems console. The imaging parameters listed in Table 1 were used to collect $\mathrm{T}_{2^{-}}$and diffusion-weighted data for each brain. For both acquisition methods, image resolution was isotropic with $0.5 \times 0.5 \times 0.5 \mathrm{~mm}$ voxels (with the exception of $0.35 \times 0.35 \times 0.35 \mathrm{~mm}$ voxels for brain E90). Slices were acquired in an interleaved manner to avoid signal loss associated with imperfections in slice-selection excitation profiles. Diffusion data were stored to disk in blocks of two transient acquisitions, and difference images from pairs of blocks were visually inspected to confirm system and sample stability during the long acquisitions. $\mathrm{T}_{2}$-weighted images were acquired before diffusion measurements to provide several hours for the sample temperature to equilibrate within the magnet bore $\left(\sim 18^{\circ} \mathrm{C}\right)$. The diffusion-sensitization gradient pulse pair consisted of duration $\delta$ of $5 \mathrm{~ms}$, interpulse interval $\Delta$ of $50 \mathrm{~ms}$, and magnitude $G$ ranging from 0 to $38 \mathrm{G} / \mathrm{cm}$. Diffusion anisotropy was measured using a 25-direction, icosahedral sampling scheme (Batchelor et al., 2003). Each $b$ value was unique in both direction and amplitude, with amplitudes ranging from 0.5 to $12.5 \mathrm{~ms} / \mu \mathrm{m}^{2}$ in equal steps of $0.5 \mathrm{~ms} / \mu \mathrm{m}^{2}$. To minimize bias in the sampling scheme, the 25 directions were sorted by proximity to an arbitrary direction and categorized into five groups of similar direction. Members of each of the five groups were assigned $b$ values from each quintile of the overall range.

Postacquisition analysis. Diffusion anisotropy was quantified in terms of relative anisotropy (RA) as defined by Basser and Pierpaoli (1996). To generate RA parameter maps, phase-sensitive images were reconstructed for each of the 25 diffusion sensitization settings. Diffusion parameters were then estimated from the 25 signal amplitudes for each voxel using a previously described "modified DTI" model selection algorithm (Kroenke et al., 2006) implemented with Bayesian Analysis software (http://bayesiananalysis.wustl.edu).

For each of the brains, RA was examined relative to the distance from the cortical surface. To do this, the apparent diffusion coefficient (ADC; defined as one third the trace of the diffusion tensor) and RA parameter maps were used to classify voxels as fixative (ADC $>$ threshold), brain (ADC $<$ threshold and RA $>0$ ), and background (the remaining voxels). The ADC threshold value was individually adjusted for each brain within the range of $0.7-0.9 \mu \mathrm{m}^{2} / \mathrm{ms}$. After this initial classification, the threedimensional (3D) volumes were visually inspected for regions interior to the brain classified as fixative, such as voxels inside ventricles. These regions were reassigned to the brain voxel class using a semiautomated, region-growing algorithm. Distances were then calculated from each brain voxel to the closest fixative voxel. The distance to the cortical surface was approximated as the intervoxel distance minus half of a voxel 
thickness, as the cortical surface is assumed to course along the border between brain and fixative voxels. Because of the discrete nature of the MRI volume data, there are 42 unique distances in the range from 0 to 3 $\mathrm{mm}(0-2.1 \mathrm{~mm}$ for the higher-resolution E90 data). Brain voxels were sorted according to distance from the brain surface. Mean RA values were determined for each of the 42 distances. Manipulation of 3D parameter maps was performed using Matlab (Mathworks, Natick, MA).

Cortical surface generation and analysis. Caret software (http:// brainmap.wustl.edu/caret) (Van Essen et al., 2001) was used to generate a surface through the cortical midthickness for brains aged E90 to E146. The standard input for segmenting adult cortex using the SureFit method in Caret is a $\mathrm{T}_{1}$-weighted brain image in which white matter has higher signal intensity than cortical gray matter, which in turn has higher signal intensity than CSF. Because of the "inverted" contrast properties of immature primate brains, the desired contrast pattern was approximately achieved by setting the grayscale image intensities of the fixative voxels of $\mathrm{T}_{2}$-weighted images (classified as described above) to zero. These adjusted $\mathrm{T}_{2}$-weighted images were used to generate an initial segmentation containing voxels "inside" or "outside" the cortical midthickness. The initial segmentation was extensively edited manually, to better match the underlying anatomy. The final segmentation was used to generate a representation of the cortical surface (Van Essen et al., 2001). For each brain, surface-based analyses were performed on the hemisphere with the least amount of damage incurred during postmortem surgical extraction. Thus, left hemisphere surfaces were generated for E90, E125a, and E125b; however, they are presented as right hemispheres (by mirror-flipping the horizontal axis) to facilitate comparisons across individuals.

In medial temporal regions, the boundary of allocortical structures (hippocampus, prepyriform cortex, periamygdaloid cortex) was difficult to delineate because it is not visible on structural MRI. The parahippocampal gyrus crown was used as the medial boundary. At the inferior medial wall boundary, where allocortex merges with gray matter nuclei such as the amygdala, a region of gray matter was identified as "unassigned" and excluded from the analysis (see Fig. $1 g$ ). This was done to avoid erroneously including subcortical structures in the cortical surface model.

Cortical diffusion anisotropy was mapped onto each surface model by assigning to each surface node the maximal RA value observed within a $(1.5 \mathrm{~mm})^{3}$ cube (or $(1.05 \mathrm{~mm})^{3}$ cube for brain E90) centered on the node. To analyze the RA pattern at E125 relative to distance from the most rapidly maturing location on the insula, the geodesic distance (shortest distance within the cortical surface) was computed between each surface node and a reference node using a function in Caret. The reference node was placed in a standard location in the anterior insula in each E125 hemisphere.

Surface-based registration and interanimal comparisons. To perform interanimal comparisons, the E146 surface was treated as a target atlas surface, and each of the other cortical surfaces was registered to the E146 target using landmark-based, surface-based registration (Van Essen, 2005). First, a "spherical standard" surface was generated for each hemisphere (with distortions reduced by multiresolution morphing followed by alignment to a standard orientation). Each individual spherical surface was registered to the E146 atlas spherical surface using the set of age-specific geographic (gyral and sulcal) landmarks listed in Table 2. Nine landmark contours were used to register the E125 hemispheres. These were chosen on the grounds that they were consistently identifiable in all three E125 hemispheres and in the E146 hemisphere and are considered likely to represent geographically and functionally corresponding locations in each individual (see Discussion). For the E90 hemisphere, we used four of these landmarks that could be delineated with confidence, plus additional landmarks along the frontal convexity and medial wall. This registration process establishes a set of point-to-point correspondences between the "standard-mesh" atlas surface (Saad et al., 2004) and the individual hemisphere surface. These were used to generate standardmesh representations of each individual surface. The three standardmesh surfaces from age E125 (E125a-E125c) were averaged to obtain an age-specific average fiducial surface.

To evaluate whether there were regional differences in the rate of expansion of cortical surface area, we computed the ratio of surface area
Table 2. Anatomical landmarks used for registration to the E146 surface

\begin{tabular}{|c|c|c|c|}
\hline Landmark & $\mathrm{E} 90$ to $\mathrm{E} 146^{a}$ & E125 to E146 & Macaque to E146 \\
\hline Dorsal medial wall & $X$ & & \\
\hline Ventral medial wall & $X$ & & \\
\hline Principal sulcus & & & $X$ \\
\hline Arcuate sulcus & & $X$ & $X$ \\
\hline Central sulcus & & $X$ & $X$ \\
\hline Sylvian fissure & $X$ & $X$ & $X$ \\
\hline Intraparietal sulcus & & $X$ & $X$ \\
\hline $\begin{array}{l}\text { Superior temporal } \\
\text { sulcus }\end{array}$ & $X$ & $X$ & $X$ \\
\hline Lunate sulcus & $x$ & $x$ & $x$ \\
\hline Cingulate sulcus & & $X$ & $X$ \\
\hline Calcarine sulcus & $X$ & $X$ & $X$ \\
\hline Inferior occipital sulcus & & & $X$ \\
\hline $\begin{array}{l}\text { Occipitotemporal } \\
\text { sulcus }\end{array}$ & & $X$ & $X$ \\
\hline Frontal convexity & $X$ & & \\
\hline
\end{tabular}

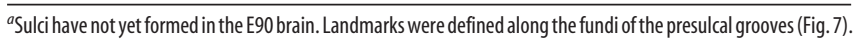

on pairs of standard-mesh fiducial surfaces at different ages. The areal expansion maps between each E125 hemisphere and the E90 hemisphere were averaged, yielding a map of early (E90-E125) expansion. Similarly, the areal expansion maps between the E146 hemisphere and each E125 hemisphere were averaged, yielding a map of late (E125-E146) expansion

To relate the diffusion anisotropy patterns observed in the baboon to cortical partitioning schemes identified in the macaque monkey, we registered the E146 baboon hemisphere to the F99 macaque surface-based atlas (available through http://sumsdb.wustl.edu/sums/directory. do?dir_id $=6585200$ ) using the landmarks specified in Table 2. Because the younger-age baboon hemispheres were also registered to the E146 hemisphere, the macaque atlas data could also be viewed on standardmesh surfaces at all ages.

The RA parametric maps, and cortical surface models generated in this study are publicly available on the SumsDB web site (http://sumsdb. wustl.edu/sums/directory.do?id $=6606357 \&$ dir_name $=$ KROENKE_ EtAl_2007).

Statistical analysis. Spearman's rank correlation coefficients were calculated to assess the relationship between RA and geodesic distance from the insula. Additionally, a linear regression in which slope and intercept parameters were estimated for RA values versus geodesic distance was compared with a modified three-parameter regression. In the threeparameter regression, slope and intercept parameters were determined for voxels corresponding to nonprimary visual and motor areas. The third parameter was the difference in intercept values between V1 motor areas and other areas. Significance in the improvement in fit of the threeparameter model over the two-parameter model was assessed using $F$ statistics. In these analyses, each voxel in the RA map was regarded as an independent measurement. This is justified because no interpolation or other transformation that affects voxel intensity values had been performed on the parametric maps. Any voxel that represented the maximal RA value for multiple nearby surface nodes contributed only a single datum to this analysis, and the associated geodesic distance from the insula was based on the average for the contributing surface nodes.

Histological analysis. Five additional brains (not included in the MRI analysis) were processed histologically. Procedures for sectioning into 5-mm-thick coronal blocks, paraffin embedding, slicing, and hematoxylin and eosin (H\&E), staining have been published previously for brains of 125, 140, and $160 \mathrm{~d}$ gestation (Dieni et al., 2004). Two additional brains of 90 and 185 d gestation were processed using identical procedures. Cortical thickness was estimated for each of the 5 brains (Dieni et al., 2004). Cortical thickness at other ages (e.g., E146 and E175) was estimated by linear interpolation between the two closest, age-matched younger and older brains. To compare cortical thickness estimates made within paraffin-embedded tissue to MRI data obtained before embedding, a correction factor was determined from E90 and E185 brains that 


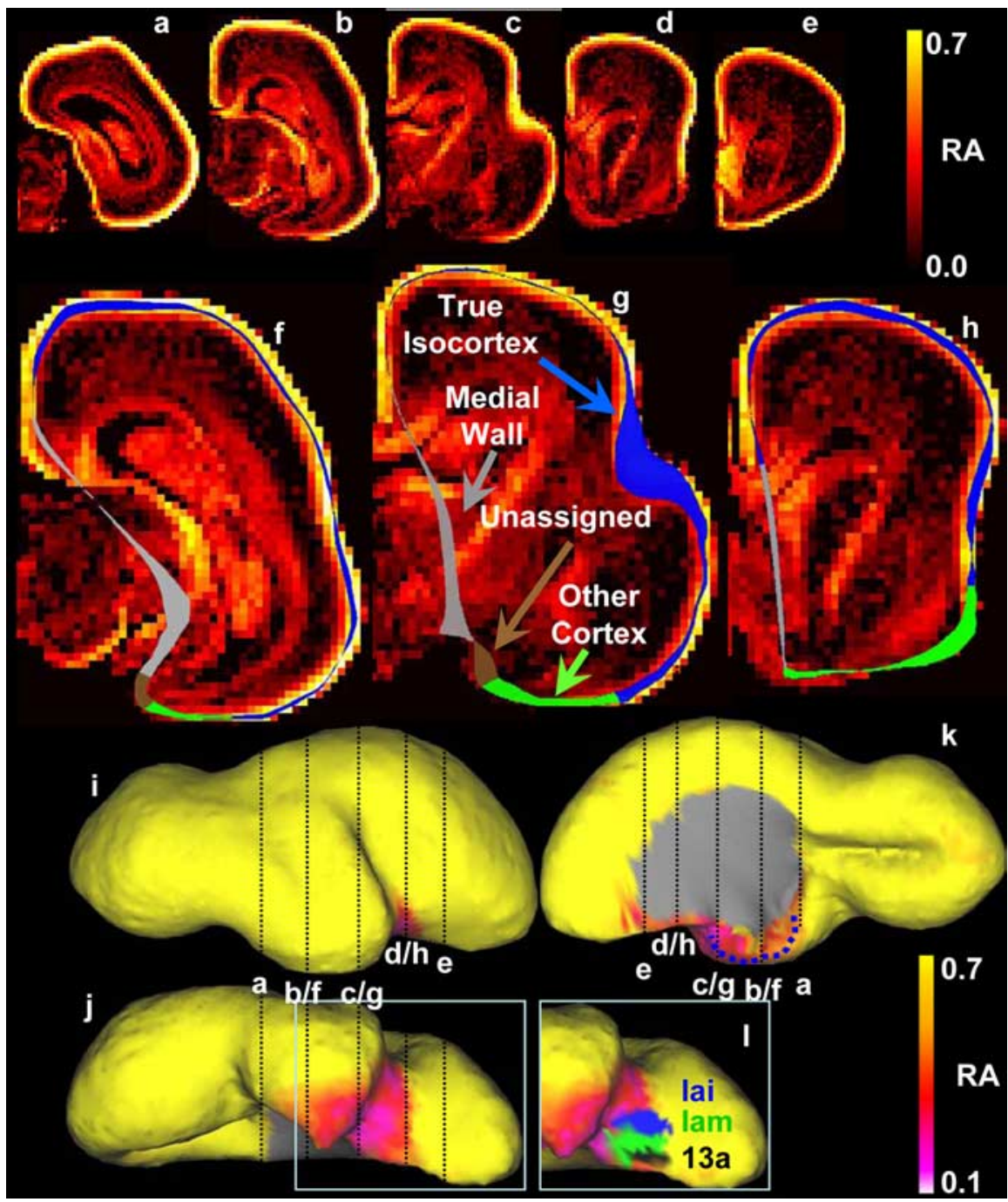

Figure 1. Isocortical versus allocortical diffusion anisotropy at E90. $\boldsymbol{a}-\boldsymbol{k}$, Coronal slices $(\boldsymbol{a}-\boldsymbol{h})$ of RA maps are shown for locations indicated on the surface models $(\boldsymbol{i}, \boldsymbol{j}, \boldsymbol{k})$. Coronal slices $\boldsymbol{f}-\boldsymbol{h}$ are enlargements of slices $\boldsymbol{b}-\boldsymbol{d}$ to show detail. The classification of surface structures used is illustrated on slices $\boldsymbol{f}-\boldsymbol{h}$. Isocortex is shown in blue, "other cortex" (including allocortex) in green, unassigned cortex in brown, and the "medial wall" (not containing cortex) in gray. In $\boldsymbol{f}-\boldsymbol{h}$, a thicker cortical surface ribbon indicates greater cortical curvature in the through-plane direction. The anisotropy color scale for "volume data" ( $\boldsymbol{a}-\boldsymbol{h})$ differs from the surface anisotropy color scale (i-I); however, in both cases, yellow is most anisotropic. At the location of the isocortex/ allocortex boundary, a dramatic change in cortical diffusion anisotropy (high-isocortex/low-allocortex) is observed. In the ventral view inset $(\boldsymbol{I})$, a color code is used to show the location of proisocortical areas lai, lam, and 13a described by Ferry et al. (2000) using surface registrations to the adult macaque atlas surface (for details, see Results, Cortical diffusion anisotropy at E90).

underwent both histological and MRI analyses. The inferior/superior extent of a coronal slice located $40 \%$ caudal to the rostral pole was measured by both methods. For both brains, formalin-immersed tissue (used for MRI) was found to be 1.3-fold larger than paraffin-embedded tissue (used for histology).

\section{Results}

Cortical diffusion anisotropy at E90: allocortex/periallocortex versus isocortex

At the earliest age examined (E90), cortical convolutions are only rudimentary, as seen in Figure 1. The Sylvian fissure (SF) and calcarine sulcus are represented by minor indentations, and most sulci present in the adult brain are absent altogether. Maps of RA derived from an E90 brain are shown in coronal slice views from caudal (Fig. 1a) to rostral (Fig. 1e). In the most caudal and rostral slices illustrated, RA values are uniformly high (yellow; RA $>0.6$ ). In intermediate slices (Figs. $1 b-d$ ), RA values are uniformly high dorsally and laterally, but there is a sharp transition to much lower values $(\mathrm{RA}<0.3)$ in a restricted region ventrally and medially, near the junction between frontal and temporal lobes.

The cortical surface of the E90 hemisphere intersected with the middle three coronal image slices in the second row of Figure 1 ( $f-h$, enlarged slices) shows the region of low anisotropy in ventromedial cortex to include classical allocortex plus adjoining periallocortical regions. Based on comparisons with architectonic subdivisions of the adult macaque (Carmichael and Price, 1994), the low-RA regions in periallocortical cortex extend into agranular insular portions of orbitofrontal cortex [areas Iam and Iai in the study by Ferry et al. (2000)] (Fig. 1l). The change from high to low anisotropy therefore appears to reflect the boundary between isocortex, defined as cortex destined to form six layers at maturity, and other cortical regions (proisocortex, periallocortex, and allocortex).

\section{Cortical diffusion anisotropy at E125:} regional variation within isocortex At age E125, cortical folding has progressed substantially, and RA is reduced overall, but in a regionally heterogeneous pattern. This is shown in axial (Fig. $2 a$ ) and coronal views (Fig. $2 b, c$ ) of RA maps for brain E125b. Lateral (Fig. 2d) and medial (Fig. 2e) views of the cortical surface model show the complete RA map. Comparison to the E90 surface model shows that many cortical sulci first appear between E90 and E125. White arrows in Figure $2 c$ delineate borders between the isocortex, other cortical structures (proisocortex, periallocortex, and allocortex), and the unassigned region. Asterisks indicate the boundaries of the proisocortex, periallocortex, and allocortex region. The extent of the unassigned region is shown between the blue dotted line and the medial wall in Figure $2 e$.

Along with an overall reduction in RA, regional heterogeneity is widespread in the E125 isocortex (contrast yellow and red arrows in Fig. 2a). The highest RA values (yellow) are in lateral occipitotemporal cortex, inferior and caudal to the superior temporal sulcus (STS). Low RA values (purple and red) are prominent in more rostral regions, including the superior temporal gyrus, the SF, the precentral gyrus (PrCG), the rostral wall of the postcentral gyrus (PoCG), cingulate gyrus, and primary visual area.

\section{Cortical diffusion anisotropy at E146}

By E146, sulci present at E125 have become substantially deeper (e.g., the cingulate sulcus), and additional sulci have appeared (e.g., the inferior occipital sulcus). The map of diffusion anisotropy is shown for lateral and medial views of the E146 surface in 
Figure 3. RA is substantially lower on average throughout the isocortex at E146 compared with E125. Nonuniformities in $\mathrm{RA}$ are less pronounced at this age than at E125, but RA values for ventral temporal cortex remain somewhat higher than for other regions.

\section{Laminar variation in cortical diffusion anisotropy}

The voxel dimensions used in this study (0.35 $\mathrm{mm}$ for E90, $0.5 \mathrm{~mm}$ for later ages) were smaller than the total cortical thickness, thereby permitting an analysis of how RA depends on depth below the pial surface. In the slice views shown in Figures 1 and 2, anisotropy tends to be highest close to the pial surface and, in some regions (Figs. 1e, $2 a$, blue arrow), shows a progressive decrease within what is likely to be the thickness of the cortical sheet. To address this issue quantitatively, the mean value of anisotropy at a given distance from the pial surface, mean RA, was plotted as a function of depth from the pial surface for six gestational ages (Fig. 4a). The pattern for brains of $146 \mathrm{~d}$ gestational age or less (Fig. $4 a$, top three panels) consists of a steep rise to a peak value from $\sim 0$ to $0.4 \mathrm{~mm}$ cortical depth, with a drop in mean RA between 0.4 and $2.0 \mathrm{~mm}$ (depending on gestational age) to a value of 0.15. The E175 and E185 brains differ from E146 and younger brains by lacking a distinct local mean RA maximum near the cortical surface.

Independent estimates of the cortical thickness at $\sim 15$ places throughout the isocortex (depending on the size of the brain) at multiple stages of gestation were made from $\mathrm{H} \& \mathrm{E}$ stained material (Dieni et al., 2004). H\&E stained sections from the superior parietal lobe are shown in Figure $4 b$. Uncorrected thickness estimates are represented as dashed vertical lines in Figure $4 a$. Because of tissue shrinkage during embedding and staining procedures, H\&Ebased measurements underestimate the cortical thickness and are thus regarded as lower limits when compared with data from the MRI experiments. Comparisons of brain dimensions determined by MRI and histology were used to calculate corrected cortical thicknesses for E90 and E185. In both cases, the MRI cortical thickness (Fig. $4 a$, solid lines) was estimated to be $30 \%$ larger than the H\&E-based measurement. These measurements demonstrate an intracortical gradient in mean RA from $0.4 \mathrm{~mm}$ to deeper layers for brains E90, E125, and E146.

\section{Diffusion anisotropy versus developmental age}

The relationship between baboon gestational age and RA is shown in Figure 4c. Note that there is a steady decline in RA with increasing gestational age. Peak RA values from Figure $4 a$ (Fig. $4 c$, filled circles) were fitted to a second order polynomial to guide the eye (solid line). Human cerebral cortical diffusion anisotropy measurements [gray triangles, taken from the study by McKinstry et al. (2002)] were registered to the baboon-derived data by scaling the baboon peak RA values to human RA. The human RA was 0.27 of the baboon peak RA. The abscissa of the plot in Figure $4 c$ relates cortical anisotropy changes as the percentage of a 40 week human gestation, assuming equivalence between a $160 \mathrm{~d}$ gestation baboon and a term human (Dieni et al., 2004).

\section{Interindividual consistency in regional isocortical} heterogeneity at E125: correspondence with isocortical maturation gradients and functional modalities

To evaluate whether the pattern of regional heterogeneity in diffusion anisotropy at E125 was consistent across individuals, we analyzed RA maps in hemispheres from three individuals (Fig. $5 a-c$ ). In the medial view of Figure $5 d$, a strip of cortex near the occipital pole is labeled gray (along with the medial wall) to indicate a region omitted from analysis because these voxels were not included in the MR images of brain E125c. Similar but not identical patterns of regional variation in RA were evident throughout the isocortex of each hemisphere. Generally, anisotropy was lowest near the insula and highest in the inferior temporal lobe.

One potential source of regional cortical anisotropy variation is a global maturation gradient throughout the isocortex. A global gradient in cortical maturation has been suggested from histolog- 

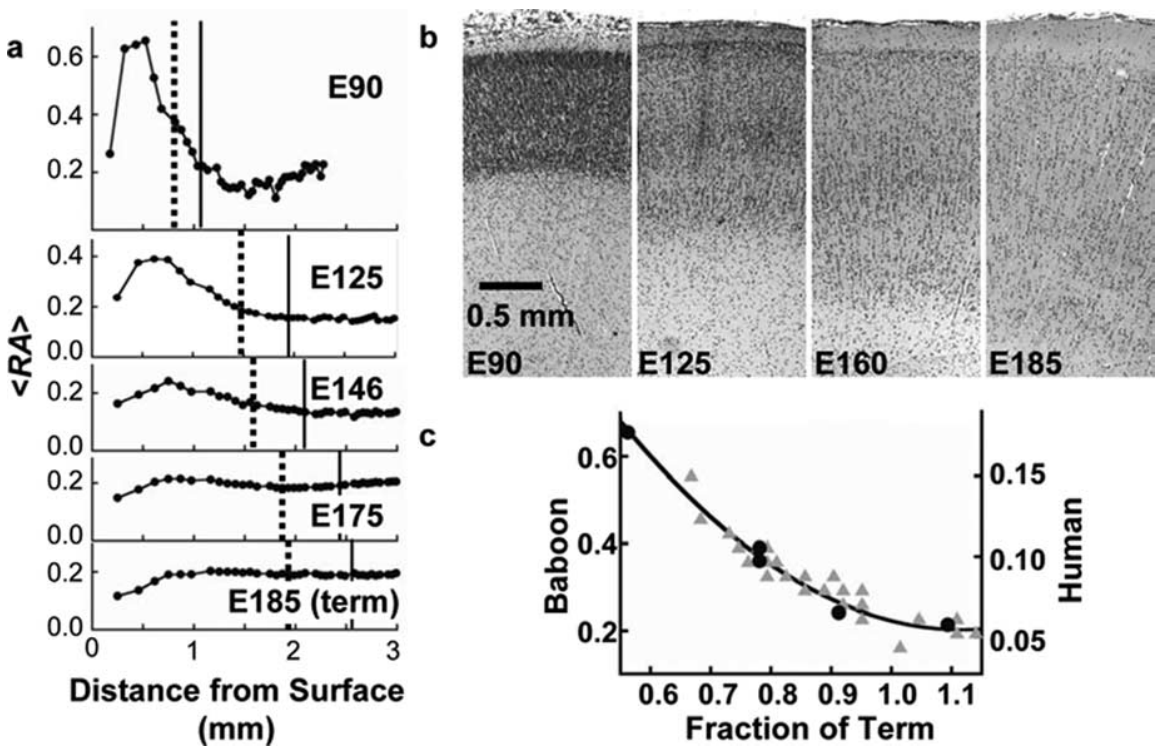

Figure 4. Relative anisotropy versus cortical depth. $\boldsymbol{a}$, The mean $\mathrm{RA}(<\mathrm{RA}>)$ is plotted versus depth from the brain surface. Cortical thickness was estimated from H\&E stained sections of brains ranging in age from E90 to E185, and is represented here as dashed vertical lines. To account for postfixation shrinkage that occurs in the paraffin embedding procedure, a correction factor of 1.3 was determined by comparing MRI data to H\&E stained E90 and E185 brains. Vertical solid lines indicate the corrected cortical thickness estimates at each age. $\boldsymbol{b}$, H\&E stained micrographs of cortical cross sections from the parietal lobe between E90 and term (E185). c, Change in baboon peak RA values (solid circles) with gestational age. The values were fitted to a polynomial to guide the eye (solid line). Data from living prematurely delivered human infants (McKinstry et al., 2002) are also shown (gray triangles).
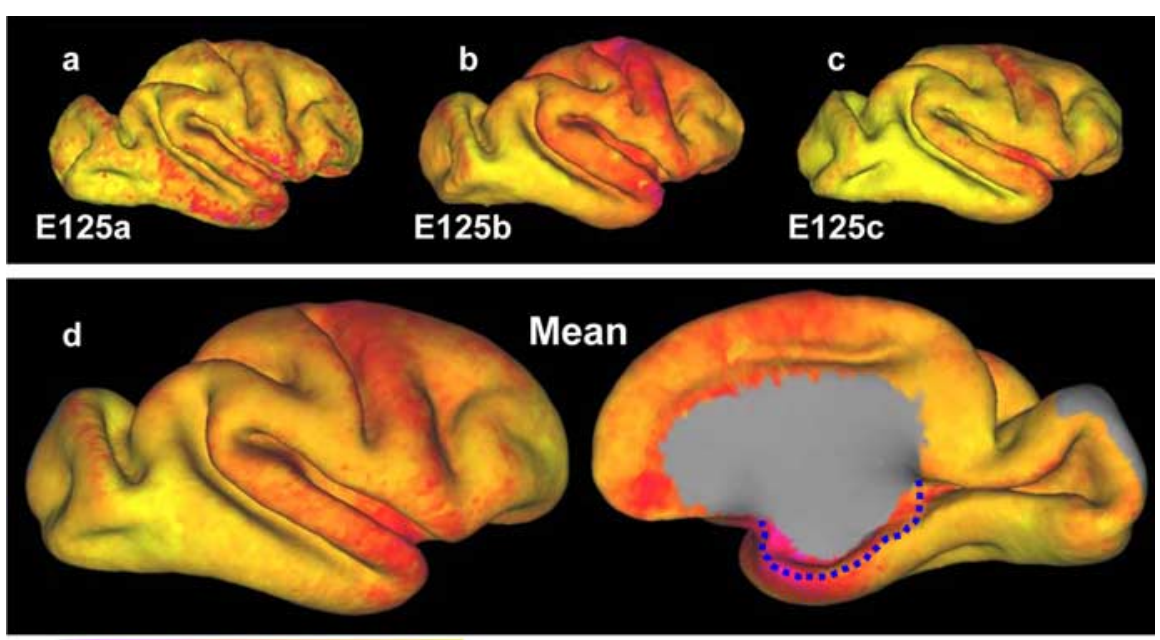

\section{1}

RA

0.7

Figure 5. Intersubject variability in the pattern of isocortical diffusion anisotropy at E125. $\boldsymbol{a}-\boldsymbol{c}$, The top row displays RA for the three E125 surfaces. In $\boldsymbol{d}$, the mean RA value from the three brains is projected onto the average E125 surface. Anisotropy values could not be measured for brain E125c in the occipital gray patch shown in the medial view (d) because of issues with RF homogeneity. This region was omitted from analysis. The dotted blue line indicates the medial extent of the parahippocampal gyrus. shown for E125b in Figure $6 b$ (solid line) and for all three brains in Figure $6 c$. Individual variability in the pattern of maturation is reflected in differences between the three lines in Figure $6 c$. The largest overall anisotropy (least mature brain) is observed in E125a; E125c is intermediate and has the steepest slope; E125b exhibits the lowest overall anisotropy and, thus, the most advanced microstructural development.

To investigate the potential influence of microstructural differences related to functional modality on diffusion anisotropy, seven functionally distinct domains were defined as shown in Figure $6 d$ : V1 (pink), somatosensory (green), motor (purple), auditory (red), olfactory (brown, not visible in the lateral view), extrastriate visual areas excluding V1 (blue), and emotion/cognition (yellow). Boundaries of these seven domains were delineated on the macaque F99 surface atlas (Van Essen, 2004), to which the baboon E146 atlas was registered using the surface landmarks listed in Table 2. In Figure 6d, the seven functional modalities are projected onto the E125b surface.

Minor differences in RA values, most of which were statistically insignificant, were observed between any pair of the seven functional domains. However, V1 and motor areas both exhibit reduced anisotropy relative to the remaining isocortex, despite the marked differences in their cytoarchitectonic characteristics. A covariate analysis was undertaken to compare RA values within these areas to others after correction for geodesic distance from the insula (Fig. 6e,f, supplemental Fig. 1, available at www.jneurosci.org as supplemental material). For each of the three brains, $F$ statistics are highly in favor $(p<0.001)$ of a model in which RA within V1 and motor areas is offset relative to remaining areas, assuming a simple linear dependence on geodesic distance. The offset linear dependence is illustrated in Figure $6 f$ (for brains E125a and E125c, see supplemental Fig. 1, available at www.jneurosci.org as supplemental material). The offset values for all three brains are listed in Table 3. ical studies (Sidman and Rakic, 1982; Smart, 1983), in which areas near the insula differentiate earlier than more distal areas. To investigate this possibility, the geodesic distance along the cortical surface from a point in the insula was calculated for each surface node. Figure $6 a$ shows the insula point location (near periallocortex) and geodesic distances from it for brain E125b. RA was plotted versus geodesic distance in Figure 6b. Spearman's rank correlation coefficients reveal a highly significant relationship $(p<0.001)$ between RA and distance from the insula in all three E125 brains (Table 3). For illustration, linear regressions are

\section{Regional patterns of cortical expansion}

The availability of accurate surface reconstructions at different developmental ages allowed us to examine global and regional aspects of the increases in surface area from E90-E146. Figure 7, $a, b, d$, and $e$, shows fiducial surfaces for the E90, average E125, and E146 hemispheres (same scale). Total hemispheric surface area increased fivefold between E90 and E146 (excluding noncortical regions along the medial wall). More specifically, surface area was $7.1 \mathrm{~cm}^{2}$ in the E90 hemisphere, $18 \mathrm{~cm}^{2}$ at E125 $(20,18$, and $16 \mathrm{~cm}^{2}$, respectively for the hemispheres in Fig. $\left.5 a-c\right)(\sim 2.5$ - 
fold greater), and $37 \mathrm{~cm}^{2}$ in the E146 hemisphere (an additional twofold greater).

Visual comparison between the E90 hemisphere and the hemispheres from later stages of maturation (E125 and E146) (Fig. 7) suggests that cortical expansion between the two ages did not occur uniformly. Surface area measurements performed on each brain revealed that the region superior and rostral to the superior temporal gyrus and lunate sulcus comprises $37 \%$ of the E90 brain, compared with an average $44 \%$ of the E125 and E146 brains $(43,44,45$, and $42 \%$ for E125a, E125b, E125c, and E146, respectively). The boundary used to delineate rostral and caudal regions is shown as a black dotted line in Figure 7. The noncortical medial wall (gray areas in Fig. 7) was excluded from the calculations of rostral and total surface areas. This difference indicates that the rostral aspect of the cortical surface expands at a faster rate than caudal regions from E90 to E125.

To characterize the regional pattern of cortical expansion in greater detail, we computed the rates of surface area expansion between each E125 fiducial surface relative to the E90 surface, using surface-based registration constrained by the landmarks displayed on each surface to generate standard-mesh representations of each hemisphere. The resulting surface area expansion map was smoothed using a standard function in Caret with default settings. The average expansion rate between E90 and E125 is displayed on lateral and medial views of a very inflated surface (Fig. $7 c$ ). Regions of relatively high expansion rate $(>0.1$ -

fold per day; yellow and orange) include medial occipital and parietal cortex and dorsolateral frontal cortex, all regions where the E146 brain contains deep sulci that are absent or barely present at E90. Regions of relatively slow expansion $(<0.05$-fold per day; red) include rostral cingulate cortex and ventral occipitotemporal cortex, where the changes in folding were less pronounced.

A similar quantitative analysis was performed between E125 and E146, using registration landmarks displayed in Figure 7, $d$ and $e$. The average expansion ratio between E125 and E146 (Fig. $7 f$ ) reveals a somewhat different regional pattern than for the earlier age comparison, including relatively fast expansion (approaching 0.15-fold per day; yellow) in medial occipital and lateral parietal cortex plus the Sylvian fissure. Regions of relatively slow expansion $(<0.1$-fold per day) include the orbitofrontal cortex and lateral occipitotemporal cortex. Although the details of this pattern depend on the choice of geographic landmarks, these results suggest that cortical expansion does not occur uniformly across the cortical sheet and that the pattern of differential expansion changes between early (E90 to E125) and late (E125 to E146) stages of maturation.

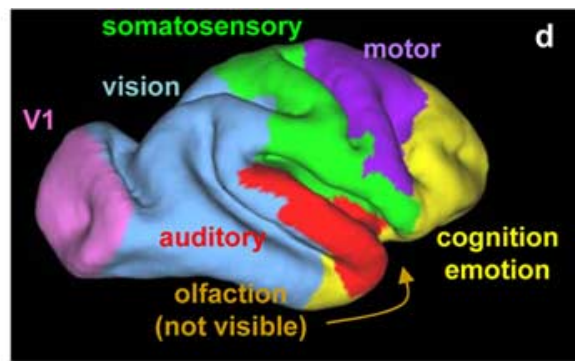

d
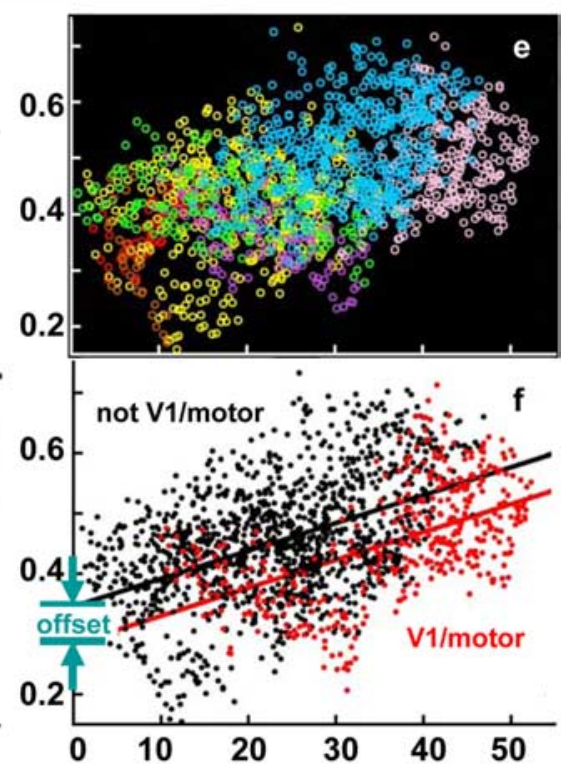

Geodesic Distance from the Insula $(\mathrm{mm})$

Figure 6. Regional variation in cortical diffusion anisotropy atE125. $\boldsymbol{a}$, The geodesic distance from a surface node on the rostral border of the insula (green asterisk) is displayed for brain E125b. Relative anisotropy values obtained from 3412 unique voxels are plotted versus geodesic distance for corresponding nodes in $\boldsymbol{b}$, and a linear fit to the data is shown to guide the eye. Similarly in motor voxels are modeled with one intercept, and V1 motor voxels are modeled with a different intercept. The "offset" parameter listed in Table 3 is the difference between intercepts illustrated in $\boldsymbol{f}$.

Table 3. Covariance of RA with distance from the insula and cortical area

\begin{tabular}{llr}
\hline Brain & $r^{a}$ & Offset $^{b}$ \\
\hline E125a & 0.16 & -0.040 \\
E125b & 0.43 & -0.062 \\
E125c & 0.47 & -0.037
\end{tabular}

${ }^{a}$ Spearman's rank correlation coefficient. High significance $(p<0.0001)$ was achieved for each brain, determined via permutation tests and analysis of $t$ statistics.

${ }^{b}$ This term is defined in the legend of Figure 6.

\section{Discussion}

Water diffusion within the immature cerebral cortex is markedly anisotropic, comparable in magnitude to diffusion within commissural white matter of the adult brain (Shimony et al., 1999). This is attributable to the highly organized, radially oriented cellular processes within the isocortex at early stages, such as occurs in the E90 baboon brain (Dieni et al., 2004). Subsequently, differentiation of cortical neurons, expansion of the neuropil, and disassembly of radial glia cells result in a loss of the prominent radial structure within the cortex and a reduction in diffusion anisotropy with gestational age (McKinstry et al., 2002). The data presented in this study of the immature baboon cortex (1) confirm the decrease in RA within the cerebral cortex with develop- 


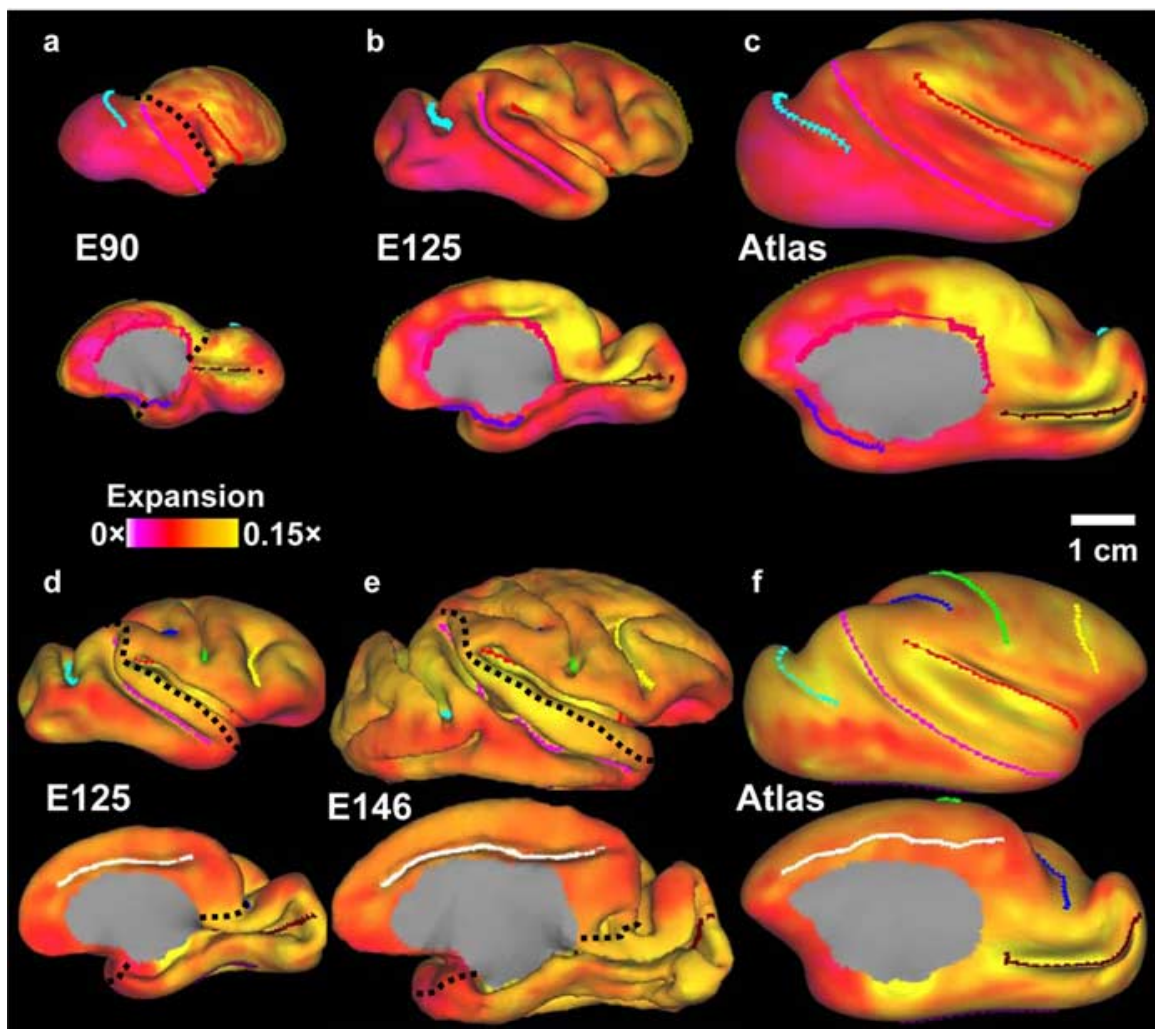

Figure 7. Regional patterns of areal expansion (the ratio of older brain surface area/younger brain surface area) divided by the age difference in days) from E90 to E125 (top two rows), and from E125 to E146 (bottom two rows). $\boldsymbol{a}$, Lateral and medial views of $\mathrm{E} 90$ standard-mesh fiducial surface with seven registration landmarks displayed. $\boldsymbol{b}$, Lateral and medial views of the average E125 standard-mesh fiducial surface with the corresponding seven registration landmarks displayed. c, Rate of areal expansion between $\mathrm{E} 90$ and E125 (mean of E125a, E125b, E125 c ratios relative to E90), displayed on a very inflated surface. Above-average expansion rates are evident in medial occipital and parietal cortex and dorsolateral frontal cortex; below-average expansion rates are evident in rostral cingulate cortex and ventral occipitotemporal cortex. $\boldsymbol{d}$, Lateral and medial views of the average E125 standard-mesh fiducial surface with nine landmark contours for registration. $\boldsymbol{e}$, Lateral and medial views of E146 standard-mesh fiducial surface, with the corresponding nine landmarks displayed. $f$, Areal expansion rates between E125 and E146 (mean of E146 relative to E125a, E125b, E125c ratios), displayed on a very inflated surface. The dotted black curve in $\boldsymbol{a}, \boldsymbol{d}$, and $\boldsymbol{e}$ shows the boundary used to calculate the percentage cortical surface area rostral to the superior temporal gyrus and lunate sulcus.

subiculum, parasubiculum, and entorhinal cortex of the hippocampal gyrus) and proisocortex (such as the agranular areas of the insula) separate "true" six-layered isocortex from allocortex. Regions of the cortex destined to differentiate into sixlayered isocortex display higher cortical diffusion anisotropy in superficial layers than these cortical areas. This contrast is most striking at E90.

\section{Regional gradients in isocortical} proliferation and differentiation

Within the developing isocortex, a spatial gradient exists in neuronal genesis and differentiation. Areas near the insula are most developmentally advanced, and more distant areas undergo progressively more delayed development. This gradient is documented in autoradiographic neuronal birth dating studies of the occipital lobe of carnivores and primates (Rakic, 1976; Luskin and Shatz, 1985; Jackson et al., 1989), examination of cortical thickness and nuclear density throughout the mouse isocortex (Smart, 1983) and occipital lobe of macaque (Smart et al., 2002), and in Golgi studies of cortical differentiation in postmortem human brain (Conel, 1939; Sidman and Rakic, 1982). Diffusion tensor imaging studies of postmortem developing mouse brain (Zhang et al., 2003) and living premature human infants (Deipolyi et al., 2005) have reported patterns of cortical diffusion anisotropy consistent with a cortical maturation gradient. The mean cortical RA map generated from the collection of E125 brains in Figure $5 d$ demonstrates that such a gradient can be observed in cortical diffusion anisotropy measure-

ment, (2) quantify regional heterogeneity in RA and cortical expansion in terms of variation distinguishing allocortex and periallocortex from isocortex, variation related to a maturation gradient originating in the insula, and an offset for primary cortical regions associated with earlier white matter development, and (3) describe differences in RA between superficial and deep cortical layers. Because of the limited number of brains evaluated in this study, variation between hemispheres and animal sex could not be evaluated. Despite this limitation, the findings described here were strikingly consistent.

These findings provide a framework for analytical methods in evaluating cortical gray matter development that have direct applicability to the human preterm infant.

\section{Regional patterns in diffusion anisotropy}

Regional variation in anisotropy was compared with three structural characteristics of the cerebral cortex.

\section{Isocortex versus other cortical regions}

The boundary between regions of high and low anisotropy shown in Figure 1 coincides with the transition region between isocortex and allocortex. The six-layer architectonic structure characteristic of isocortex differs from the more primitive allocortex (Zilles, 2004). Intermediate zones of periallocortex (including the pre- ments. Anisotropy is lower (cortex more mature) within isocortical areas near the insula and central sulcus, and higher (cortex less mature) at the more distal frontal pole and inferior temporal region (Fig. 6).

\section{Primary/early myelin-forming versus nonprimary/late} myelin-forming areas

Modest but statistically significant differences between primary visual and motor cortices were observed after correction for geodesic distance from the insula. These two areas contribute and receive projections from the early myelinating posterior limb of the internal capsule (primary motor), and optic radiations (primary visual) (Brody et al., 1987; Kinney et al., 1988; Kostovic et al., 2002; Kizildag et al., 2005). It is therefore plausible that early myelination of axonal tracts occurs in concert with early cortical differentiation relative to neighboring areas. In addition to their potentially distinct developmental tempo, primary visual and motor cortices also differ from the rest of isocortex in their adult cytoarchitecture (Zilles, 2004). A distinguishing feature of primary visual cortex is a prominent layer IV, consisting of a dense dendritic network. In contrast, primary motor cortex possesses a notably sparse layer IV plus large pyramidal neurons (Betz cells) in layer V. It is interesting that both of these departures from "typical" cortical structure correlate with reduced cortical diffu- 
sion anisotropy. Additional experiments are necessary to determine whether the distinctive anisotropy pattern observed for primary visual and motor cortices is related to specific aspects of development, architecture at maturity, or both.

\section{Diffusion anisotropy throughout the cortical depth}

Anisotropy is highest in superficial layers of isocortex, with the exception of layer I, which is thinner than the dimensions of MRI voxels used in this study. Although the rudiments of a six-layered adult laminar structure can be seen in E125 brains (Dieni et al., 2004), the immature isocortex of E90 and E125 brains do not yet possess a mature, differentiated morphology (Fig. 2b) (Sidman and Rakic, 1982; Coogan and Van Essen, 1996; Dieni et al., 2004). Nevertheless, immature neurons of superficial layers excluding layer I are elongated (Dieni et al., 2004), have a relatively undifferentiated neuropil [Sidman and Rakic (1982) their Figs. 1-20], and exhibit a higher degree of radial organization than more differentiated, deeper layers (Rakic, 1972). A laminar anisotropy pattern is observed in averaged profiles throughout the isocortex in Figure $4 a$, reflective of the increased cellular-level morphological organization in superficial cortical layers. Inspection of RA parameter maps further document this laminar pattern within individual slices located at several locations within the cortex at both E90 and E125 (Figs. $1 a-h, 2 a$ ). A similar dependence of anisotropy on cortical depth has also been observed in rodent studies (Sizonenko et al., 2007).

\section{Relation to patterns of cortical folding and expansion}

In considering the large regional differences in cortical expansion illustrated in Figure 7, it is important to consider two major factors that may be responsible: (1) the underlying neuroanatomical processes, and (2) any limitations in our methods that may cause artificial distortions in the observed regional pattern of surface area expansion across development. With regard to the underlying neuroanatomical processes, neurogenesis is mostly complete by E90 in the macaque (gestation period, $\sim 165 \mathrm{~d}$ ) and presumably soon thereafter in the baboon (gestation period, $185 \mathrm{~d})$. Thus, nonuniform expansion of the cortex might be related to regional differences in subsequent events such as gliogenesis, morphological differentiation of neurons and glial cells, and/or synaptogenesis. By this hypothesis, a unit cortical surface area at one age might expand more in one region than in another because it might gain in the number (or average size) of neurons, glial cells, synapses, and/or axons during the intervening period. In the macaque (and presumably also in the baboon), there is regional heterogeneity in the timing of neurogenesis, migration of cortical neurons to the cortical plate (Sidman and Rakic, 1982), and synaptogenesis (Granger et al., 1995). However, it is not obvious that the reported regional differences are pronounced enough to account for the observed heterogeneity in cortical expansion. The magnitude and underlying mechanism of differential areal expansion merits additional investigation.

With regard to methodological limitations, there are at least two processes to consider. First, the surface-based registration algorithm used to align landmarks may produce artifactual distortions. Such distortions may contribute to local fluctuations in the areal distortion maps, but it is unlikely that they are a major contributor to the large regional differences illustrated in Figure 7. Furthermore, estimates of cortical surface area rostral to the superior temporal gyrus and lunate sulcus were made for the E90-E146 brains independently of the surface registration algorithm, and these estimates also demonstrate regional variation in cortical surface expansion. A second potential methodological issue relates to sliding landmarks. Neurons located on a given gyral or sulcal crease at one age might shift physically and be positioned at a different location (e.g., in the bank of a sulcus) at a later age. This would potentially result in a false appearance of regional variation in cortical surface area expansion because of changes in the geographic features used for defining landmarks. Although this explanation would be interesting from a developmental perspective, the existing data does not provide evidence in favor or against this possibility.

\section{Comparisons to prematurely delivered human infants}

Comparisons between living and postmortem rodent brain indicate that death and fixation do not markedly alter diffusion anisotropy (Sun et al., 2003). Thus, the data obtained from fixed nonhuman primate tissue have strong relevance to studies of intact human infants. This assertion is supported by the data in Figure $4 c$, which demonstrate a striking similarity in the temporal decrease in anisotropy for developing baboon and human relative to other neurodevelopmental stages such as cortical folding and white matter myelination (Dieni et al., 2004). To date, the application of DTI methods to the premature human infant brain has been focused primarily on the detection of white matter injury (Huppi, 2004). However, some of the neurodevelopmental challenges faced by prematurely born infants may reflect injury to gray matter as well as white matter (Inder et al., 2005). Furthermore, white matter and cerebral cortex are intimately connected, making it difficult to injure one without affecting the other. Conventional imaging methods have not proven especially effective in delineating cortical injury in this patient population. As MRI systems become more sophisticated with improved technology (Dumoulin et al., 2002; Harel et al., 2006) and knowledge of normative data increases (Evans and Grp, 2006; Hermoye et al., 2006; Huang et al., 2006), cortical diffusion anisotropy measurement holds promise for investigating the developing cerebral cortex in prematurely delivered human infants.

\section{Conclusions}

MR measurements of cortical gray matter RA depict several well known features of cortical maturation. Early in development, RA values for allocortex and periallocortex are lower than those for isocortex, reflecting differences in the microarchitecture of these regions. In addition, RA values for isocortex are higher in the less mature superficial layers, consistent with differences in cortical microstructure. RA measurements provide an overview of cortical maturation with evaluation of the entire cortical surface in each subject studied. This demonstrates global decreases in cortical RA with maturation. Superimposed on this global maturation is regional heterogeneity suggesting that (1) cortical regions closer to the insula mature at a faster rate than those distant from the insula, and (2) primary visual and motor cortex differentiate at a faster rate than other areas. The concordance between these findings and those of histology-based studies serves to confirm the validity of this unique, MRI-based approach. The fact that it can be applied to living subjects makes this a potentially powerful tool for evaluating normal and disordered cortical development in animal models and human subjects.

\section{References}

Basser PJ, Pierpaoli C (1996) Microstructural and physiological features of tissues elucidated by quantitative-diffusion-tensor MRI. J Magn Reson Ser B 111:209-219.

Batchelor PG, Atkinson D, Hill DL, Calamante F, Connelly A (2003) Anisotropic noise propagation in diffusion tensor MRI sampling schemes. Magn Reson Med 49:1143-1151. 
Brody BA, Kinney HC, Kloman AS, Gilles FH (1987) Sequence of central nervous system myelination in human infancy. I. An autopsy study of myelination. J Neuropathol Exp Neurol 46:283-301.

Carmichael ST, Price JL (1994) Architectonic subdivision of the orbital and medial prefrontal cortex in the macaque monkey. J Comp Neurol 346:366-402.

Conel JL (1939) The postnatal development of the human cerebral cortex. Cambridge, MA: Harvard UP.

Coogan TA, Van Essen DC (1996) Development of connections within and between areas V1 and V2 of macaque monkeys. J Comp Neur 372:327-342.

Deipolyi AR, Mukherjee P, Gill K, Henry RG, Partridge SC, Veeraraghavan S, Jin H, Lu Y, Miller SP, Ferriero DM, Vigneron DB, Barkovich AJ (2005) Comparing microstructural and macrostructural development of the cerebral cortex in premature newborns: diffusion tensor imaging versus cortical gyration. NeuroImage 27:579-586.

Dieni S, Inder TE, Yoder B, Briscoe T, Camm E, Egan G, Denton D, Rees S (2004) The pattern of cerebral injury in a primate model of preterm birth and neonatal intensive care. J Neuropathol Exp Neurol 63:1297-1309.

Doty FD, Entzminger G, Hauck CD (1999) Error-tolerant RF litz coils for NMR/MRI. J Magn Reson 140:17-31.

Dumoulin CL, Rohling KW, Piel JE, Rossi CJ, Giaouinto RO, Watkins RD, Vigneron DB, Barkovich AJ, Newton N (2002) Magnetic resonance imaging compatible neonate incubator. Concepts Magn Reson 15:117-128.

Evans AC, Grp BD (2006) The NIH MRI study of normal brain development. NeuroImage 30:184-202.

Ferry AT, Ongur D, An X, Price JL (2000) Prefrontal cortical projections to the striatum in macaque monkeys: evidence for an organization related to prefrontal networks. J Comp Neur 425:447-470.

Granger B, Tekaia F, Le Sourd AM, Rakic P, Bourgeois J-P (1995) Tempo of neurogenesis and synaptogenesis in the primate cingulate mesocortex: comparison with the neocortex. J Comp Neur 360:363-376.

Harel N, Ugurbil K, Uludag K, Yacoub E (2006) Frontiers of brain mapping using MRI. J Mag Reson Imaging 23:945-957.

Hermoye L, Saint-Martin C, Cosnard G, Lee SK, Kim J, Nassogne MC, Menten R, Clapuyt P, Donohue PK, Hua K, Wakana S, Jiang H, van Zijl PCM, Mori S (2006) Pediatric diffusion tensor imaging: Normal database and observation of the white matter maturation in early childhood. NeuroImage 29:493-504.

Huang H, Zhang J, Wakana S, Zhang J, Ren T, Richards LJ, Yarowsky P, Donohue PK, Graham E, Van Zijl PC, Mori S (2006) White and gray matter development in human fetal, newborn and pediatric brains. NeuroImage, in press.

Huppi PS (2004) Immature white matter lesions in the premature infant. J Pediatr 145:575-578.

Huppi PS, Maier SE, Peled S, Zientara GP, Barnes PD, Jolesz FA, Volpe JJ (1998) Microstructural development of human newborn cerebral white matter assessed in vivo by diffusion tensor magnetic resonance imaging. Pediatr Res 44:584-590.

Inder TE, Warfield SK, Wang H, Huppi PS, Volpe JJ (2005) Abnormal cerebral structure is present at term in premature infants. Pediatrics 115:286-294.

Jackson CA, Peduzzi JD, Hickey TL (1989) Visual cortex development in the ferret. I. Genesis and migration of visual cortical neurons. J Neurosci 9:1242-1253.

Jespersen SN, Kroenke CD, Ostergaard L, Ackerman JJH, Yablonskiy DA (2007) Modeling dendrite density from magnetic resonance diffusion measurements. NeuroImage 34:1473-1486.

Kinney HC, Brody BA, Kloman AS, Gilles FH (1988) Sequence of central nervous system myelination in human infancy. II. Patterns of myelination in autopsied infants. J Neuropathol Exp Neurol 47:217-234.

Kizildag B, Dusunceli E, Fitoz S, Erden I (2005) The role of classic spin echo and FLAIR sequences for the evaluation of myelination in MR imaging. Diagn Intervent Radiol 11:130-136.

Kostovic I, Judas M, Rados M, Hrabac P (2002) Laminar organization of the human fetal cerebrum revealed by histochemical markers and magnetic resonance imaging. Cereb Cortex 12:536-544.
Kroenke CD, Ackerman JJ, Yablonskiy DA (2004) On the nature of the NAA diffusion attenuated MR signal in the central nervous system. Magn Reson Med 52:1052-1059.

Kroenke CD, Bretthorst GL, Inder TE, Neil JJ (2005) Diffusion MR imaging characteristics of the developing primate brain. NeuroImage 25:1205-1213.

Kroenke CD, Bretthorst GL, Inder TE, Neil JJ (2006) Modeling water diffusion anisotropy within fixed newborn primate brain using Bayesian probability theory. Magn Reson Med 55:187-197.

Luskin MB, Shatz CJ (1985) Neurogenesis of the cat's primary visual cortex. J Comp Neur 242:611-631.

McKinstry RC, Mathur A, Miller JP, Ozcan AO, Snyder AZ, Schefft GL, Almli CR, Shiran SI, Conturo TE, Neil JJ (2002) Radial organization of developing human cerebral cortex revealed by non-invasive water diffusion anisotropy MRI. Cereb Cortex 12:1237-1243.

Neil JJ, Shiran SI, McKinstry RC, Schefft GL, Snyder AZ, Almli CR, Akbudak E, Aaronovitz JA, Miller JP, Lee BCP, Conturo TE (1998) Normal brain in human newborns: apparent diffusion coefficient and diffusion anisotropy measured using diffusion tensor imaging. Radiology 209:57-66.

Rakic P (1972) Mode of cell migration to the superficial layers of fetal monkey neocortex. J Comp Neur 145:61-84.

Rakic P (1976) Differences in the time of origin and in eventual distribution of neurons in areas 17 and 18 of visual cortex in rhesus monkey. Exp Brain Res [Suppl] 1:244-248.

Rakic P (1995) A small step for the cell, a giant leap for mankind: a hypothesis of neocortical expansion during evolution. Trends Neurosci 18:383-388.

Saad ZS, Reynolds R, Argall BD, Japee S, Cox RW (2004) SUMA: an interface for surface-based intra- and inter-subject analysis with AFNI. In: Proceedings of the 2004 IEEE International Symposium on Biomedical Imaging, Arlington, VA, pp 1510-1513. New York: IEEE.

Shimony JS, McKinstry RC, Akbudak E, Aronovitz JA, Snyder AZ, Lori NF, Cull TS, Conturo TE (1999) Quantitative diffusion-tensor anisotropy brain MR imaging: Normative human data and anatomic analysis. Radiology 212:770-784.

Sidman RL, Rakic P (1982) Development of the human central nervous system. In: Histology and histopathology of the nervous system (Haymaker W, Adams RD, eds), pp 3-145. Springfield, IL: Charles C. Thomas.

Sizonenko SV, Camm EJ, Garbow JR, Maier S, Inder TE, Kiss JZ, Williams CE, Neil JJ, Huppi PS (2007) Developmental changes and injury induced disruption of the radial organisation of the cortex in the immature rat brain revealed by in vivo diffusion tensor MRI (DTI). Cereb Cortex 17:2609-2617.

Smart IH (1983) Three dimensional growth of the mouse isocortex. J Anat 137:683-694

Smart IHM, Dehay C, Biroud P, Berland M, Kennedy H (2002) Unique morphological features of the proliferative zones and postmitotic compartments of the neural epithelium giving rise to striate and extrastriate cortex in the monkey. Cereb Cortex 12:37-53.

Sun SW, Neil JJ, Song SK (2003) Relative indices of water diffusion anisotropy are equivalent in live and formalin-fixed mouse brains. Magn Reson Med 50:743-748.

Van Essen DC (2004) Surface-based approaches to spatial localization and registration in primate cerebral cortex. NeuroImage 23:S97-S107.

Van Essen DC (2005) A population-average, landmark- and surface-based (PALS) atlas of human cerebral cortex. NeuroImage 28:635-662.

Van Essen DC, Dickson J, Harwell J, Hanlon D, Anderson CH, Drury HA (2001) An integrated software system for surface-based analyses of cerebral cortex. J Am Med Informat Assoc 41:1359-1378.

Zhang J, Richards LJ, Yarowsky P, Huang H, van Zijl PCM, Mori S (2003) Three-dimensional anatomical characterization of the developing mouse brain by diffusion tensor microimaging. NeuroImage 20:1639-1648.

Zilles K (2004) Architecture of the human cerebral cortex. In: The human nervous system (Paxinos G, Mai JK, eds), pp 997-1055. San Diego: Elsevier Academic. 